\title{
Crystal structure of bis(tetrapropylammonium) tetraiodoplumbate(II), $\left(\left(\mathrm{C}_{3} \mathrm{H}_{7}\right)_{4} \mathbf{N}\right)_{2} \mathrm{PbI}_{4}$
}

\author{
M. Geselle and H. Fuess
}

Technische Hochschule Darmstadt, FB Materialwissenschaft-Strukturforschung, Petersenstr. 20, D-64287 Darmstadt, Germany

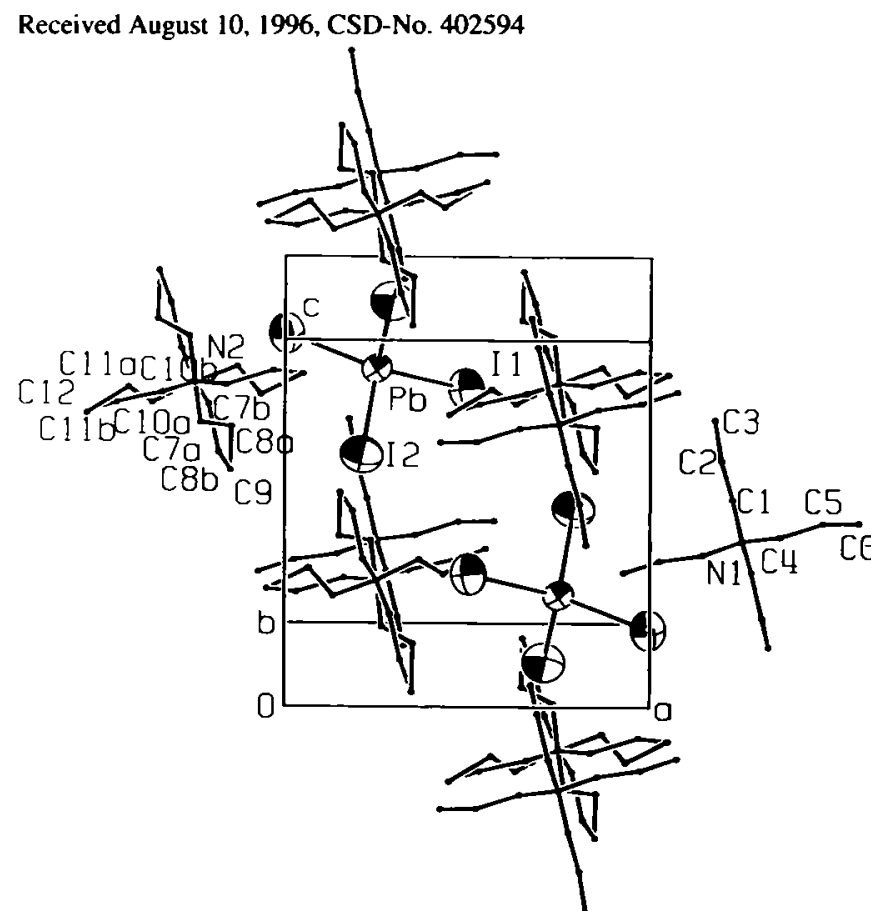

Source of material: Yellow platelets of the title compound were obtained by slow evaporation of a DMSO solution containing lead(II)iodide and tetrapropylammonium iodide in stoichiometric quantities.

The crystal structure of the title compound consists of isolated, distorted tetrahedral $\mathrm{PbI}_{4}{ }^{2-}$-anions and tetrapropylammonium cations. The packing is similar to that observed in the corresponding Co-, Zn-, and Cd-tetrahalogenometallates with tetraethylammonium as cation. In the title compound half of the cations are orientationally disordered as it is the case with the corresponding tetraethylammonium tetrahalogenometallates. Due to the presence of a lone electron pair, the distortion of the anion from the ideal tetrahedral symmetry is relatively severe.

Table 1. Parameters used for the X-ray data collection

Crystal:

Wavelength:

$\mu$ :

Diffractometer:

Scan mode:

$T_{\text {measurement: }}$

$2 \theta_{\text {max }}$ :

$\mathrm{N}(h k l)_{\text {unique: }}$

Criterion for $F_{\mathrm{o}}$ :

N(param) refined

Program: yellow platelet, size $0.175 \times 0.125 \times 0.425 \mathrm{~mm}$ Mo $K_{\alpha}$ radiation $(0.71073 \AA)$

$76.3 \mathrm{~cm}^{-1}$

Enraf-Nonius CAD4

$\theta / 2 \theta$

$297 \mathrm{~K}$

$46^{\circ}$

2006

$F_{\mathrm{o}}>2 \sigma\left(F_{\mathrm{o}}\right)$

157

$\mathrm{Xtal} 3.2$
$\mathrm{C}_{24} \mathrm{H}_{56} \mathrm{I}_{4} \mathrm{~N}_{2} \mathrm{~Pb}$, monoclinic, $P 12 / n 1$ (No. 13), $a=11.100(1) \AA, b=14.944(2) \AA, c=11.560(2) \AA, \beta=90.28(2)^{\circ}$, $V=1917.5 \AA^{3}, Z=2, R(F)=0.063, R_{w}(F)=0.045$.

Table 2. Final atomic coordinates and displacement parameters (in $\AA^{2}$ )

\begin{tabular}{|c|c|c|c|c|c|c|}
\hline Atom & Site & Occ. & $x$ & $y$ & $z$ & $U_{\text {iso }}$ \\
\hline H(1a) & $4 g$ & & $0.158(1)$ & $1.0013(9)$ & $0.327(1)$ & 0.15 \\
\hline H(lb) & $4 g$ & & $0.292(1)$ & $1.0003(9)$ & $0.361(1)$ & 0.15 \\
\hline $\mathbf{H}(2 \mathrm{a})$ & $4 g$ & & $0.121(1)$ & 0.889 (1) & $0.455(1)$ & 0.15 \\
\hline $\mathbf{H}(\mathbf{2 b})$ & $4 g$ & & $0.255(1)$ & $0.883(1)$ & $0.486(1)$ & 0.15 \\
\hline $\mathbf{H}(3 \mathbf{a})$ & $4 g$ & & $0.156(2)$ & $0.967(1)$ & $0.631(1)$ & 0.15 \\
\hline $\mathbf{H}(3 \mathbf{b})$ & $4 g$ & & $0.246(2)$ & $1.027(1)$ & $0.566(1)$ & 0.15 \\
\hline$H(3 c)$ & $4 g$ & & $0.111(2)$ & $1.032(1)$ & $0.537(1)$ & 0.15 \\
\hline$H(4 a)$ & $4 g$ & & $0.337(1)$ & $0.8067(9)$ & $0.339(1)$ & 0.15 \\
\hline H(4b) & $4 g$ & & $0.371(1)$ & $0.8061(9)$ & $0.211(1)$ & 0.15 \\
\hline H(5a) & $4 g$ & & $0.496(1)$ & $0.925(1)$ & $0.242(1)$ & 0.15 \\
\hline $\mathbf{H}(5 \mathbf{b})$ & $4 g$ & & $0.466(1)$ & $0.921(1)$ & $0.371(1)$ & 0.15 \\
\hline H(6a) & $4 g$ & & $0.646(1)$ & $0.846(1)$ & $0.345(1)$ & 0.15 \\
\hline H(6b) & $4 g$ & & $0.547(1)$ & $0.778(1)$ & $0.376(1)$ & 0.15 \\
\hline $\mathbf{H}(\mathbf{6 c )}$ & $4 g$ & & $0.586(1)$ & $0.789(1)$ & $0.250(1)$ & 0.15 \\
\hline H(7aa) & $4 g$ & 0.5 & $0.688(3)$ & $0.635(2)$ & $0.603(1)$ & 0.20 \\
\hline $\mathbf{H}(7 \mathbf{a b})$ & $4 g$ & 0.5 & $0.81(3)$ & $0.575(2)$ & $0.589(1)$ & 0.20 \\
\hline $\mathbf{H}(7 \mathrm{ba})$ & $4 g$ & 0.5 & $0.853(3)$ & $0.688(1)$ & $0.710(1)$ & 0.20 \\
\hline $\mathbf{H}(7 \mathrm{bb})$ & $4 g$ & 0.5 & $0.721(3)$ & $0.698(1)$ & $0.672(1)$ & 0.20 \\
\hline H(8ba) & $4 g$ & 0.5 & $0.753(3)$ & $0.6118(8)$ & $0.510(1)$ & 0.20 \\
\hline $\mathbf{H}(8 \mathrm{bb})$ & $4 g$ & 0.5 & $0.885(3)$ & $0.6071(8)$ & $0.547(1)$ & 0.20 \\
\hline H(8aa) & $4 g$ & 0.5 & $0.928(3)$ & $0.672(2)$ & $0.614(1)$ & 0.20 \\
\hline H(8ab) & $4 g$ & 0.5 & $0.851(3)$ & $0.755(2)$ & $0.638(1)$ & 0.20 \\
\hline $\mathrm{H}(9 \mathrm{aa})$ & $4 g$ & 0.5 & $0.869(2)$ & $0.711(1)$ & $0.4020(9)$ & 0.20 \\
\hline$H(9 a b)$ & $4 g$ & 0.5 & $0.913(2)$ & $0.759(1)$ & $0.5120(9)$ & 0.20 \\
\hline $\mathrm{H}(9 \mathrm{ac})$ & $4 g$ & 0.5 & $0.780(2)$ & $0.764(1)$ & $0.4763(9)$ & 0.20 \\
\hline$H(9 b a)$ & $4 g$ & 0.5 & $0.912(2)$ & $0.767(1)$ & $0.4626(9)$ & 0.20 \\
\hline $\mathrm{H}(9 \mathrm{bb})$ & $4 g$ & 0.5 & $0.775(2)$ & $0.754(1)$ & $0.4576(9)$ & 0.20 \\
\hline$H(9 b c)$ & $4 g$ & 0.5 & $0.860(2)$ & $0.675(1)$ & $0.4343(9)$ & 0.20 \\
\hline H(10aa) & $4 g$ & 0.5 & $0.645(2)$ & $0.539(2)$ & $0.623(2)$ & 0.20 \\
\hline$H(10 a b)$ & $4 g$ & 0.5 & $0.581(2)$ & $0.606(2)$ & $0.702(2)$ & 0.20 \\
\hline H(10ba) & $4 g$ & 0.5 & $0.840(1)$ & $0.4803(9)$ & $0.688(3)$ & 0.20 \\
\hline $\mathrm{H}(10 \mathrm{bb})$ & $4 g$ & 0.5 & $0.809(1)$ & $0.4697(9)$ & $0.817(3)$ & .0 .20 \\
\hline H(11aa) & $4 g$ & 0.5 & $0.544(2)$ & $0.496(2)$ & $0.833(2)$ & 0.20 \\
\hline $\mathbf{H}(11 \mathrm{ab})$ & $4 g$ & 0.5 & $0.883(2)$ & $0.428(2)$ & $0.732(2)$ & 0.20 \\
\hline H(11ba) & $4 g$ & 0.5 & $0.499(1)$ & $0.5685(8)$ & $0.763(3)$ & 0.20 \\
\hline H(1.lbb) & $4 g$ & 0.5 & $0.532(1)$ & $0.5624(8)$ & $0.634(3)$ & 0.20 \\
\hline$H(12 a b)$ & $4 g$ & 0.5 & $0.420(1)$ & $0.396(1)$ & $0.728(2)$ & 0.20 \\
\hline H(12aa) & $4 g$ & 0.5 & $0.470(1)$ & $0.438(1)$ & $0.616(2)$ & 0.20 \\
\hline$H(12 a c)$ & $4 g$ & 0.5 & $0.392(1)$ & $0.494(1)$ & $0.699(2)$ & 0.20 \\
\hline $\mathrm{H}(12 \mathrm{ba})$ & $4 g$ & 0.5 & $0.371(1)$ & $0.466(1)$ & $0.677(2)$ & 0.20 \\
\hline$H(12 b b)$ & $4 g$ & 0.5 & $0.452(1)$ & $0.416(1)$ & $0.764(2)$ & 0.20 \\
\hline$H(12 b c)$ & $4 g$ & 0.5 & $0.480(1)$ & $0.412(1)$ & $0.634(2)$ & 0.20 \\
\hline
\end{tabular}


Table 3. Final atomic coordinates and displacement parameters (in $\AA^{2}$ )

\begin{tabular}{|c|c|c|c|c|c|c|c|c|c|c|c|}
\hline Atom & Site & Occ. & $x$ & $y$ & $z$ & $U_{11}$ & $U_{22}$ & $U_{33}$ & $U_{12}$ & $U_{13}$ & $U_{23}$ \\
\hline $\mathbf{P b}(1)$ & $2 f$ & & $1 / 4$ & $0.75954(7)$ & $3 / 4$ & $0.0851(6)$ & $0.1049(8)$ & $0.0799(6)$ & 0 & $0.0076(4)$ & o \\
\hline I(1) & $4 g$ & & $0.4958(1)$ & $0.84320(9)$ & $0.6749(1)$ & $0.116(1)$ & $0.0977(9)$ & $0.168(1)$ & $-0.0123(8)$ & $-0.0036(9)$ & $0.0110(9)$ \\
\hline I (2) & $4 g$ & & $0.2094(1)$ & $0.64358(9)$ & $0.5407(1)$ & $0.173(1)$ & $0.108(1)$ & $0.144(1)$ & $-0.008(1)$ & $-0.006(1)$ & $-0.0264(9)$ \\
\hline $\mathbf{N}(1)$ & $2 e$ & & $1 / 4$ & $0.902(1)$ & $1 / 4$ & $0.09(1)$ & $0.049(9)$ & $0.09(1)$ & 0 & $0.011(9)$ & 0 \\
\hline$C(1)$ & $4 g$ & & $0.224(1)$ & $0.9647(9)$ & $0.349(1)$ & $0.09(1)$ & $0.07(1)$ & $0.09(1)$ & $0.001(9)$ & $0.004(8)$ & $-0.005(8)$ \\
\hline$C(2)$ & $4 g$ & & $0.193(1)$ & $0.921(1)$ & $0.464(1)$ & $0.13(1)$ & $0.10(1)$ & $0.07(1)$ & $0.02(1)$ & $0.005(9)$ & $-0.006(9)$ \\
\hline$C(3)$ & $4 g$ & & $0.174(2)$ & $0.994(1)$ & $0.559(1)$ & $0.18(2)$ & $0.13(2)$ & $0.12(1)$ & $0.02(2)$ & $0.02(1)$ & $-0.01(1)$ \\
\hline$C(4)$ & $4 g$ & & $0.357(1)$ & $0.8426(9)$ & $0.276(1)$ & $0.09(1)$ & $0.07(1)$ & $0.10(1)$ & $0.016(9)$ & $0.013(9)$ & $0.002(9)$ \\
\hline$C(5)$ & $4 g$ & & $0.475(1)$ & $0.887(1)$ & $0.303(1)$ & $0.09(1)$ & $0.10(1)$ & $0.12(1)$ & $0.01(1)$ & $0.00(1)$ & $0.00(1)$ \\
\hline$C(6)$ & $4 g$ & & $0.573(1)$ & $0.819(1)$ & $0.320(1)$ & $0.10(1)$ & $0.17(2)$ & $0.15(2)$ & $0.04(1)$ & $0.00(1)$ & $-0.03(1)$ \\
\hline $\mathbf{N}(2)$ & $2 e$ & & $3 / 4$ & $0.5834(5)$ & $3 / 4$ & $0.11(1)$ & $0.08(1)$ & $0.09(1)$ & 0 & $0.00(1)$ & 0 \\
\hline$C(7 a)$ & $4 g$ & 0.5 & $0.765(3)$ & $0.622(2)$ & $0.632(1)$ & $0.10(2)$ & $0.20(4)$ & $0.39(4)$ & $0.00(2)$ & $0.08(2)$ & $0.18(3)$ \\
\hline$C(7 b)$ & $4 g$ & 0.5 & $0.787(3)$ & $0.659(1)$ & $0.674(1)$ & $0.10(2)$ & $0.20(4)$ & $0.39(4)$ & $0.00(2)$ & $0.08(2)$ & $0.18(3)$ \\
\hline C(8a) & $4 g$ & 0.5 & $0.852(3)$ & $0.698(2)$ & $0.604(1)$ & $0.12(2)$ & $0.16(4)$ & $0.14(3)$ & $-0.02(2)$ & $-0.01(2)$ & $-0.01(2)$ \\
\hline$C(8 b)$ & $4 g$ & 0.5 & $0.816(3)$ & $0.6429(8)$ & $0.547(1)$ & $0.12(2)$ & $0.16(4)$ & $0.14(3)$ & $-0.02(2)$ & $-0.01(2)$ & $-0.01(2)$ \\
\hline$C(9)$ & $4 g$ & & $0.849(2)$ & $0.727(1)$ & $0.4779(9)$ & $0.21(2)$ & $0.35(4)$ & $0.16(2)$ & $0.00(3)$ & $0.04(2)$ & $0.14(2)$ \\
\hline$C(10 a)$ & $4 g$ & 0.5 & $0.632(2)$ & $0.556(2)$ & $0.700(2)$ & $0.09(2)$ & $0.09(3)$ & $0.17(2)$ & $0.02(1)$ & $0.03(2)$ & $0.02(2)$ \\
\hline$C(10 b)$ & $4 g$ & 0.5 & $0.838(1)$ & $0.5094(9)$ & $0.760(3)$ & $0.09(2)$ & $0.09(3)$ & $0.17(2)$ & $0.02(1)$ & $0.03(2)$ & $0.02(2)$ \\
\hline C(1la) & $4 g$ & 0.5 & $0.566(2)$ & $0.477(2)$ & $0.758(2)$ & $0.09(2)$ & $0.20(4)$ & $0.13(3)$ & $0.03(2)$ & $-0.03(2)$ & $0.01(2)$ \\
\hline$C(11 b)$ & $4 g$ & 0.5 & $0.532(1)$ & $0.5315(8)$ & $0.705(3)$ & $0.09(2)$ & $0.20(4)$ & $0.13(3)$ & $0.03(2)$ & $-0.03(2)$ & $0.01(2)$ \\
\hline$C(12)$ & $4 g$ & & $0.451(1)$ & $0.448(1)$ & $0.694(2)$ & $0.17(2)$ & $0.21(3)$ & $0.25(3)$ & $-0.09(2)$ & $-0.03(2)$ & $0.00(2)$ \\
\hline
\end{tabular}

\section{References}

1. Geselle, M.: Strukturelle Eigenschaften zweiwertiger Halogenoplumbate organischer Stickstoffbasen. PhD thesis. Technische Hochschule Darmstadt, Germany 1996.
2. Hall, S. R., Flack, H. D., Stewart, J. M.: Xtal 3.2 Reference Manual, Universities of Western Australia, Maryland, and Geneva 1992.

3. Burnett, M. N., Johnson, C. K.: ORTEP-III: Oak Ridge Thermal Ellipsoid Plot Program for Crystal Structure Illustrations, Oak Ridge National Laboratory Report ORNL-6895, USA 1996. 